\title{
Preoperative chemoradiation therapy induces primary-tumor complete response more frequently than chemotherapy alone in gastric cancer: analyses of the National Cancer Database 2006-2014 using propensity score matching
}

\author{
Naruhiko Ikoma ${ }^{1,7}$. Prajnan Das ${ }^{2} \cdot$ Wayne Hofstetter $^{3} \cdot$ Jaffer A. Ajani $^{4} \cdot$ Jeannelyn S. Estrella ${ }^{5}$ Hsiang-Chun Chen ${ }^{6}$. \\ Xuemei Wang ${ }^{6} \cdot$ Rashida A. Callender $^{7}$. Cong Zhu ${ }^{7}$. Christina L. Roland ${ }^{1} \cdot$ Keith F. Fournier $^{1} \cdot$ Janice N. Cormier ${ }^{1}$. \\ Paul Mansfield ${ }^{1} \cdot$ Brian D. Badgwell $^{1}$
}

Received: 27 February 2018 / Accepted: 27 April 2018 / Published online: 5 May 2018

(c) The International Gastric Cancer Association and The Japanese Gastric Cancer Association 2018

\begin{abstract}
Background The benefit of preoperative chemoradiation (CXRT) over preoperative chemotherapy alone ("chemotherapy" hereafter) is unknown. By analyzing the National Cancer Database (NCDB), we investigated whether preoperative CXRT improves the incidence of primary tumor pathologic complete response (ypT0) and overall survival (OS) compared with preoperative chemotherapy in patients with gastric cancer.

Methods Patients with non-metastatic gastric adenocarcinoma who underwent CXRT or chemotherapy followed by gastrectomy were included. Propensity score matching with a ratio of 1:1 was implemented to reduce selection bias. A conditional logistic regression model was used to compare incidences of ypT0 between groups, and Cox proportional hazards model was used to compare OS.

Results We identified 8464 patients. Median patient age was 63 years; $76 \%$ were male and $79 \%$ were white. ypT0 was observed in $16.1 \%$ of patients in the CXRT group and $6.6 \%$ in the chemotherapy group $(p<0.001)$. After propensity score matching, a total of 2408 patients were matched. CXRT was associated with a higher incidence of ypT0 (OR 2.28, 95\% CI $1.76-2.95 ; p<0.0001$ ) and higher frequency of R0 resection (92 vs. 86\%; $p<0.001$ ). However, CXRT was not associated with longer OS (HR 1.03, 95\% CI $0.92-1.15 ; p=0.63$ ). Safety profiles (30-day mortality, 30-day readmission, and length of hospital stay) were equivalent between groups.

Conclusions In this study of gastric cancer patients from the NCDB, CXRT was associated with a higher incidence of ypT0 and $\mathrm{R} 0$ resection compared with chemotherapy, although it was not associated with a longer OS.
\end{abstract}

Keywords Gastric cancer $\cdot$ Preoperative chemoradiation therapy $\cdot$ Complete response $\cdot$ Surgery $\cdot$ Survival

This manuscript was presented as an oral presentation at 2017

Annual Meeting of the Western Surgical Association (Nov. 6th).

Brian D. Badgwell

bbadgwell@mdanderson.org

1 Department of Surgical Oncology, The University of Texas MD Anderson Cancer Center, 1400 Pressler Street, Fct17.6010, Houston, TX 77030, USA

2 Department of Radiation Oncology, The University of Texas MD Anderson Cancer Center, Houston, TX, USA

3 Department of Cardiac and Thoracic Surgery, The University of Texas MD Anderson Cancer Center, Houston, TX, USA

4 Department of Gastrointestinal Medical Oncology, The University of Texas MD Anderson Cancer Center, Houston, TX, USA
5 Department of Pathology, The University of Texas MD Anderson Cancer Center, Houston, TX, USA

6 Department of Biostatistics, The University of Texas MD Anderson Cancer Center, Houston, TX, USA

7 Department of Biostatistics, School of Public Health, The University of Texas Health Science Center at Houston, Houston, TX, USA 


\section{Background}

Over the past two decades, treatment strategies for gastric cancer have undergone a paradigm shift, from surgical resection alone to multimodality therapy. To identify the ideal treatment approach for resectable gastric cancers, several randomized studies have been conducted and have reported a significant survival benefit from postoperative therapies $[1,2]$ and from preoperative or perioperative therapies [3, 4] compared with surgery alone. Most notably, after the MAGIC trial showed a survival benefit associated with perioperative chemotherapy, the use of preoperative chemotherapy significantly increased in the United States over the last 10 years [3-6]. However, the survival benefit of preoperative chemoradiation (CXRT) over preoperative chemotherapy alone ("chemotherapy" hereafter) is unknown and is currently under investigation in an international randomized controlled trial (the TOPGEAR trial) [7]. Preliminary results of the TOPGEAR trial reported equivalent safety of preoperative CXRT compared with chemotherapy, as well as a high proportion of treatment completion in the CXRT group; however, the authors did not report the difference in pathologic response between groups to maintain equipoise [7]. In advance of the reporting of these level one data, analysis of the effectiveness of CXRT for resectable gastric cancer using a large, hospital-based database is helpful to inform current treatment for patients with resectable gastric cancer. Therefore, we conducted a retrospective cohort study, by analyzing the National Cancer Database (NCDB), to investigate whether preoperative CXRT improves the incidence of ypT0 and overall survival (OS) compared with preoperative chemotherapy in patients with gastric cancer.

\section{Methods}

\section{Data source}

Data from the NCDB, provided by the Commission on Cancer $(\mathrm{CoC})$ of the American College of Surgeons and the American Cancer Society, were analyzed [8]. Data were collected from $\mathrm{CoC}$-accredited cancer program registries using nationally standardized data items and coding definitions. Approximately, $70 \%$ of all newly diagnosed cases of cancer in the United States are reported to the NCDB [8].

\section{Inclusion and exclusion criteria}

In total, 168,377 patients with gastric tumor (International Classification of Diseases for oncology, codes C16.0 to C16.9) were reported to the NCDB between 2004 and 2014.
Because the treatment sequence code was available only after 2006, we did not include patients with diagnoses during the years 2004-2005. Patients with non-metastatic gastric adenocarcinoma [tumor histology codes 8140 (adenocarcinoma, NOS), 8144 (adenocarcinoma, intestinal type), 8145 (adenocarcinoma, diffuse type), 8481 (mucin-producing adenocarcinoma), and 8490 (signet ring cell adenocarcinoma); tumor behavior 3 (invasive); clinical stage M0] who underwent preoperative chemotherapy followed by gastrectomy [surgical procedure code 30-80 (gastrectomy, NOS; neartotal or total gastrectomy; gastrectomy, NOS with removal of a portion of esophagus; and gastrectomy with resection in continuity with the resection of other organs, NOS); systemic therapy surgery sequence code 2 (systemic therapy given before surgery) or 4 (systemic therapy given before and after surgery)] were included in the study cohort. To improve the validity of the study, we did not include patients with time between the date of chemotherapy initiation and date of surgery less than 30 days or longer than 360 days. For the study objectives, patients who did not have pathologic T stage (pT stage) information were not included.

\section{Variables}

The primary outcome measure for the study was ypT0, defined as pT category 0 (no residual tumor in the primary site), and the main exposure was the regimen of preoperative therapy (CXRT vs. chemotherapy). CXRT was defined as the use of preoperative radiation therapy [surgery-radiation sequence code 2 (radiation given before surgery) or 4 (radiation given before and after surgery)], excluding patients with radiation dose recorded as 0 or a radiation therapy target (volume) coded as other than 12 (esophagus), 13 (stomach), 17 (abdomen, NOS), or 98/99 (others/unknown). Other covariates included age, race, sex, clinical $\mathrm{T}$ stage (American Joint Committee on Cancer [AJCC] TNM staging system, 8th edition [9]), tumor location, hospital setting, year of diagnosis, duration of preoperative chemotherapy (defined as time between the date of chemotherapy initiation and date of surgery), type of radiation therapy (conventional or advanced technique; defined as use of intensity-modulated radiation therapy, 3-D conformal radiation therapy, or proton therapy), and dose of preoperative radiation therapy.

\section{Statistical analysis}

Patient characteristics were summarized overall and stratified by preoperative therapy regimen using descriptive statistics. Differences between categorical variables were compared using Fisher's exact tests or Chi square tests, and differences between continuous variables were compared using two-sided Student's $t$ test, as appropriate. Univariate and multivariate logistic regression models were applied to 
assess the relationship between the patient characteristics and ypT0. Overall survival was defined as the time interval between date of surgery and date of death, with OS censored at the last follow-up date for patients who were alive. Survival curves were estimated using the Kaplan-Meier method. Univariate and multivariate Cox proportional hazards regression models were fit to assess the association between patient characteristics and OS. Patient characteristics that were significant in the univariate model at the 0.10 level were included in the multivariate model. Then, backward elimination was implemented until all remaining predictors had a $p$ value less than 0.05 . Preoperative therapy regimen was forced to stay in the model. Covariates were transformed as appropriate [10].

Propensity score matching was implemented to reduce the possibility of selection bias on preoperative radiation therapy. The Greedy $8 \rightarrow 1$ digit match algorithm was applied in propensity score matching. Age, race, sex, clinical $\mathrm{T}$ stage, tumor location, hospital setting, and year of diagnosis were used as the matching criteria to estimate the propensity scores. Patients who received CXRT were matched to patients who received chemotherapy with a ratio of $1: 1$. Only patients with known vital status were included in the matching process. Matching was performed using the SAS macro oneToManyMTCH [11]. The standardized differences were used to evaluate the balance of patient characteristics between treatment groups before and after propensity score adjustment. After adjustment, Cox proportional hazards models with robust variance estimator were applied to assess the association between patient characteristics and OS. Conditional logistic regression analyses on matched data were used to evaluate the association between patient characteristics and the incidence of ypT0. As secondary analyses, the safety profile (post-surgical hospital stay length, frequency of 30-day mortality, and frequency of readmission within 30 days), frequency of R0 resection (negative surgical margin), and number of lymph nodes examined by the pathologist were compared between the two groups using McNemar's test and two-sided Student's $t$ test, as appropriate. All statistical analyses were performed using SAS 9.3 (SAS Institute, Cary, NC) and Stata 14.1 (Stata Corp, College Station, TX, USA).

\section{Results}

We identified 8464 patients with gastric cancer who underwent preoperative CXRT or chemotherapy followed by gastrectomy. Table 1 summarizes the patient characteristics, both overall and stratified by preoperative therapy regimen, before and after propensity score matching. The median patient age was 63 years; $76 \%$ were male and $79 \%$ were white. There were significant differences in age, race, sex, clinical T stage, tumor location, hospital setting, and year of diagnosis between groups. After propensity score matching, a total of 2408 patients (1204 in each group) were matched. Most notably, because of high proportions of white race $(90.8 \%)$ and cardia tumor location $(95.1 \%)$ in CXRT group, matched cohorts are mostly representing white $83.7 \%$ in CXRT group and $83.2 \%$ in chemotherapy group) and cardia tumor ( $87.5 \%$ in both CXRT group and chemotherapy group). All absolute values of standardized differences were less than $10 \%$, indicating that they were well-balanced (Table 1).

\section{Complete response in the primary tumor (урт0)}

In this study, $983(11.6 \%)$ of 8464 patients had ypT0, including $16.1 \%$ [721/4488; 95\% confidence interval (CI), 15.0-17.2\%] of patients who received CXRT and 6.6\% (262/3976; 95\% CI 5.8-7.4\%) of patients who received chemotherapy $(p<0.001)$. Of the 983 patients with ypT0, $85 \%(602 / 709 ; 12$ patients with unknown pathologic $N$ category) who received CXRT had ypN0 (true complete response) along with $84 \%(215 / 256 ; 6$ patients with unknown pathologic $N$ category) of those who received chemotherapy $(p=0.919)$. Table 2 presents the results of univariate and multivariate logistic regression models of ypT0. By multivariable logistic regression, CXRT was associated with a higher incidence of ypT0 [odds ratio (OR) 2.6, 95\% CI 2.21-3.06; $p<0.001]$. Age, sex, clinical T stage, hospital setting, and duration between chemotherapy and surgery also remained significant in the final model. By the conditional logistic regression analyses for matched cohort, the receipt of preoperative CXRT was the only variable associated with a higher incidence of ypT0 by univariate analysis. For reference, a multivariate model with the same variables used in the model for unmatched cohort was fit to the matched cohort; preoperative CXRT remained a significant predictor for ypT0 (OR 2.28, 95\% CI 1.76-2.95; $p<0.0001$ ).

\section{Overall survival}

Among 6888 patients with known vital status, 3646 (53\%) had died and $3242(47 \%)$ were alive. The median follow-up time among survivors was 31.4 months (range 0.05-113.4). The median OS was 30.8 months (95\% CI 29.3-32.3). Table 3 presents the results of univariate and multivariate Cox proportional hazards models for OS. Age, race, sex, clinical T stage, tumor location, hospital setting, year of diagnosis, preoperative therapy regimen (Fig. 1a), and duration between chemotherapy and surgery were associated with OS in the univariate analysis and were included in the multivariate model. By multivariate analysis, CXRT was not associated with improved OS [hazard ratio (HR) 1.05, 95\% CI $0.96-1.15 ; p=0.33$ ]. 
Table 1 Patient characteristics

\begin{tabular}{|c|c|c|c|c|c|c|c|c|}
\hline \multirow{3}{*}{$\overline{\text { Age }-N(\%)}$} & \multirow{2}{*}{$\begin{array}{l}\text { All patients } \\
(N=8464)\end{array}$} & \multicolumn{4}{|l|}{ Before matching } & \multicolumn{3}{|l|}{ After matching } \\
\hline & & $\begin{array}{l}\text { With radiation } \\
(N=4488)\end{array}$ & $\begin{array}{l}\text { Without } \\
\text { radiation } \\
(N=3976)\end{array}$ & $p$ & Std diff $(\%)$ & $\begin{array}{l}\text { With radiation } \\
(N=1204)\end{array}$ & $\begin{array}{l}\text { Without } \\
\text { radiation } \\
(N=1204)\end{array}$ & Std diff $(\%)$ \\
\hline & & & & $<0.001$ & 18.01 & & & 4.48 \\
\hline$<50$ & $1179(13.9 \%)$ & $522(11.6 \%)$ & $657(16.5 \%)$ & & & $146(12.1 \%)$ & $164(13.6 \%)$ & \\
\hline $50-69$ & $5113(60.4 \%)$ & $2883(64.2 \%)$ & $2230(56.1 \%)$ & & & $750(62.3 \%)$ & $739(61.4 \%)$ & \\
\hline$\geq 70$ & $2172(25.7 \%)$ & $1083(24.1 \%)$ & $1089(27.4 \%)$ & & & $308(25.6 \%)$ & $301(25 \%)$ & \\
\hline Race $-N(\%)$ & & & & $<0.001$ & 64.33 & & & 4.02 \\
\hline White & $6682(78.9 \%)$ & $4076(90.8 \%)$ & $2606(65.5 \%)$ & & & $1008(83.7 \%)$ & $1002(83.2 \%)$ & \\
\hline Black & $783(9.3 \%)$ & $190(4.2 \%)$ & $593(14.9 \%)$ & & & $78(6.5 \%)$ & $85(7.1 \%)$ & \\
\hline Hispanic & $648(7.7 \%)$ & $149(3.3 \%)$ & $499(12.6 \%)$ & & & $79(6.6 \%)$ & $84(7 \%)$ & \\
\hline Asian/other & $351(4.1 \%)$ & $73(1.6 \%)$ & $278(7 \%)$ & & & $39(3.2 \%)$ & $33(2.7 \%)$ & \\
\hline $\mathrm{Sex}-N(\%)$ & & & & $<0.001$ & 64.28 & & & 1.34 \\
\hline Male & $6391(75.5 \%)$ & $3748(83.5 \%)$ & $2643(66.5 \%)$ & & & $956(79.4 \%)$ & $952(79.1 \%)$ & \\
\hline Female & $2073(24.5 \%)$ & $740(16.5 \%)$ & $1333(33.5 \%)$ & & & $248(20.6 \%)$ & $252(20.9 \%)$ & \\
\hline $\begin{array}{l}\text { Clinical T } \\
\text { stage }-N(\%), \\
N=7204\end{array}$ & & & & $<0.001$ & 33.44 & & & 4.30 \\
\hline $0 / 1$ & $546(7.6 \%)$ & $237(5.9 \%)$ & $309(9.6 \%)$ & & & $93(7.7 \%)$ & $100(8.3 \%)$ & \\
\hline 2 & $1442(20 \%)$ & $740(18.6 \%)$ & $702(21.8 \%)$ & & & $271(22.5 \%)$ & $266(22.1 \%)$ & \\
\hline 3 & $4797(66.6 \%)$ & $2884(72.4 \%)$ & $1913(59.4 \%)$ & & & $770(64 \%)$ & $778(64.6 \%)$ & \\
\hline $4 \mathrm{a}$ & $354(4.9 \%)$ & $114(2.9 \%)$ & $240(7.5 \%)$ & & & $64(5.3 \%)$ & $55(4.6 \%)$ & \\
\hline $4 \mathrm{~b}$ & $65(0.9 \%)$ & $11(0.3 \%)$ & $54(1.7 \%)$ & & & $6(0.5 \%)$ & $5(0.4 \%)$ & \\
\hline $\begin{array}{l}\text { Tumor loca- } \\
\text { tion }-N(\%) \text {, } \\
N=8037\end{array}$ & & & & $<0.001$ & 135.58 & & & 6.00 \\
\hline Cardia & $5772(71.8 \%)$ & $4213(95.1 \%)$ & $1559(43.2 \%)$ & & & $1054(87.5 \%)$ & $1054(87.5 \%)$ & \\
\hline Fundus/body & $1001(12.5 \%)$ & $95(2.1 \%)$ & $906(25.1 \%)$ & & & $64(5.3 \%)$ & $73(6.1 \%)$ & \\
\hline Antrum/pylorus & $836(10.4 \%)$ & $69(1.6 \%)$ & 767 (21.3\%) & & & $49(4.1 \%)$ & $50(4.2 \%)$ & \\
\hline Overlapping & $428(5.3 \%)$ & $55(1.2 \%)$ & $373(10.3 \%)$ & & & $37(3.1 \%)$ & $27(2.2 \%)$ & \\
\hline $\begin{array}{l}\text { Hospital } \\
\text { setting-N(\%) }\end{array}$ & & & & $<0.001$ & 7.76 & & & 5.38 \\
\hline Community & $426(5 \%)$ & $241(5.4 \%)$ & $185(4.7 \%)$ & & & $62(5.1 \%)$ & $55(4.6 \%)$ & \\
\hline Comprehensive & $2387(28.2 \%)$ & $1320(29.4 \%)$ & 1067 (26.8\%) & & & $306(25.4 \%)$ & $291(24.2 \%)$ & \\
\hline Academic & $4422(52.2 \%)$ & $2313(51.5 \%)$ & $2109(53 \%)$ & & & $689(57.2 \%)$ & $694(57.6 \%)$ & \\
\hline Other & $1229(14.5 \%)$ & $614(13.7 \%)$ & $615(15.5 \%)$ & & & $147(12.2 \%)$ & $164(13.6 \%)$ & \\
\hline $\begin{array}{l}\text { Year of diagno- } \\
\text { sis- } N(\%)\end{array}$ & & & & 0.01 & 3.29 & & & 2.70 \\
\hline 2006-2010 & $3141(37.1 \%)$ & $1606(35.8 \%)$ & $1535(38.6 \%)$ & & & $593(49.3 \%)$ & $574(47.7 \%)$ & \\
\hline 2011-2014 & $5323(62.9 \%)$ & $2882(64.2 \%)$ & $2441(61.4 \%)$ & & & $611(50.7 \%)$ & $630(52.3 \%)$ & \\
\hline
\end{tabular}

Std diff standardized difference

$p$ values less than 0.05 were bolded

After propensity score matching, 1279 (53\%) of 2408 matched patients had died and $1129(47 \%)$ were alive at last follow-up. The median OS was 32 months (95\% CI 29.2-35 months). Sex, clinical T stage, and tumor location were significantly associated with OS in the univariate analysis. CXRT was not significantly associated with OS (Fig. 1b). By multivariate analysis, sex, clinical $\mathrm{T}$ stage, and tumor location remained significant in the multivariate model, and CXRT was not associated with improved OS (HR $1.03,95 \%$ CI $0.92-1.15 ; p=0.63$ ). 
Table 2 Logistic regression analyses for complete response

\begin{tabular}{|c|c|c|c|c|}
\hline \multirow[t]{2}{*}{ Variable } & \multicolumn{2}{|c|}{$\begin{array}{l}\text { Unmatched } \\
983 \text { pts had CR in } 8464 \text { pts }\end{array}$} & \multicolumn{2}{|c|}{$\begin{array}{l}\text { Matched } \\
327 \text { pts had CR in } 2408 \text { pts }\end{array}$} \\
\hline & $\mathrm{OR}(95 \% \mathrm{CI})$ & $p$ & $\mathrm{OR}(95 \% \mathrm{CI})$ & $p$ \\
\hline \multicolumn{5}{|l|}{ Univariate } \\
\hline \multicolumn{5}{|l|}{ Age } \\
\hline $50-69($ ref. $<50)$ & $1.66(1.32-2.1)$ & \multirow[t]{2}{*}{$<0.001$} & $0.82(0.28-2.43)$ & \multirow[t]{2}{*}{0.67} \\
\hline$\geq 70($ ref. $<50)$ & $1.75(1.37-2.25)$ & & $0.54(0.13-2.18)$ & \\
\hline \multicolumn{5}{|l|}{ Race } \\
\hline Black (ref. white) & $0.6(0.45-0.78)$ & \multirow[t]{3}{*}{$<0.001$} & $0.24(0.05-1.2)$ & \multirow[t]{3}{*}{0.31} \\
\hline Hispanic (ref. white) & $0.57(0.42-0.76)$ & & $1.23(0.15-10.31)$ & \\
\hline Asian/other (ref. white) & $0.6(0.41-0.89)$ & & $0.56(0.06-5.74)$ & \\
\hline \multicolumn{5}{|l|}{ Sex } \\
\hline Female (ref. male) & $0.66(0.55-0.78)$ & $<0.001$ & $0.46(0.16-1.31)$ & 0.14 \\
\hline \multicolumn{5}{|l|}{ Clinical T stage } \\
\hline 2 (ref. 1) & $0.89(0.67-1.17)$ & \multirow[t]{3}{*}{0.01} & $1.2(0.29-4.99)$ & \multirow[t]{3}{*}{0.74} \\
\hline 3 (ref. 1) & $0.79(0.62-1.02)$ & & $0.61(0.16-2.3)$ & \\
\hline $4 \mathrm{a} / 4 \mathrm{~b}$ (ref. 1) & $0.49(0.32-0.75)$ & & $0.75(0.16-3.41)$ & \\
\hline \multicolumn{5}{|l|}{ Hospital setting } \\
\hline Comprehensive (ref. community) & $1.13(0.81-1.58)$ & \multirow[t]{3}{*}{0.001} & $0.56(0.12-2.75)$ & \multirow[t]{3}{*}{0.68} \\
\hline Academic (ref. community) & $1.26(0.91-1.74)$ & & $0.93(0.18-4.98)$ & \\
\hline Other (ref. community) & $0.8(0.55-1.16)$ & & $0.47(0.09-2.38)$ & \\
\hline \multicolumn{5}{|l|}{ Year of diagnosis } \\
\hline 2011-2014 (ref. 2006-2010) & $1.23(1.07-1.41)$ & 0.004 & $0.8(0.32-2.03)$ & 0.64 \\
\hline \multicolumn{5}{|l|}{ Preoperative therapy } \\
\hline CXRT (ref. chemotherapy) & $2.71(2.34-3.15)$ & $<0.001$ & $2.21(1.72-2.84)$ & $<0.001$ \\
\hline \multicolumn{5}{|l|}{ Radiation dose } \\
\hline 40-60 Gy (ref. <40 Gy) & $1.08(0.79-1.48)$ & 0.64 & $0.87(0.35-2.19)$ & 0.25 \\
\hline \multicolumn{5}{|l|}{ Radiation type } \\
\hline Advanced (ref. conventional) & $1.07(0.91-1.26)$ & 0.40 & $1.09(0.64-1.85)$ & 0.04 \\
\hline Duration between chemo and surger & & & & \\
\hline $60-89($ ref. $<60)$ & $1.71(1.07-2.73)$ & 0.004 & $0.86(0.25-2.9)$ & 0.99 \\
\hline 90-119 (ref. <60) & $1.96(1.23-3.12)$ & & $0.82(0.25-2.72)$ & \\
\hline$\geq 120($ ref. $<60)$ & $2.12(1.32-3.4)$ & & $0.83(0.25-2.8)$ & \\
\hline Multivariate & & & & \\
\hline Age & & & & \\
\hline $50-69($ ref. $<50)$ & $1.38(1.08-1.76)$ & 0.01 & $0.57(0.12-2.72)$ & 0.53 \\
\hline$\geq 70($ ref. $<50)$ & $1.55(1.19-2.03)$ & & $0.34(0.05-2.25)$ & \\
\hline Sex & & & & \\
\hline Female vs. male & $0.82(0.69-0.99)$ & 0.04 & $0.25(0.05-1.4)$ & 0.12 \\
\hline Clinical T stage & & & & \\
\hline Female (ref. male) & $0.81(0.61-1.08)$ & 0.002 & $0.63(0.08-5.29)$ & 0.64 \\
\hline 2 (ref. 1) & $0.67(0.52-0.86)$ & & $0.43(0.07-2.84)$ & \\
\hline 3 (ref. 1) & $0.56(0.36-0.86)$ & & $0.29(0.03-2.83)$ & \\
\hline Hospital setting & & & & \\
\hline Comprehensive (ref. community) & $1.08(0.75-1.55)$ & 0.02 & $0.55(0.08-4.03)$ & 0.52 \\
\hline Academic (ref. community) & $1.22(0.86-1.73)$ & & $0.89(0.08-9.53)$ & \\
\hline Other (ref. community) & $0.85(0.57-1.27)$ & & $0.23(0.02-2.29)$ & \\
\hline Preoperative therapy & & & & \\
\hline CXRT (ref. chemotherapy) & $2.6(2.21-3.06)$ & $<0.001$ & $2.28(1.76-2.95)$ & $<0.001$ \\
\hline Duration between chemo and surger & & & & \\
\hline $60-89($ ref. $<60)$ & $1.31(0.78-2.19)$ & 0.003 & $0.7(0.17-2.89)$ & 0.85 \\
\hline 90-119 (ref. <60) & $1.71(1.02-2.84)$ & & $0.76(0.19-3.07)$ & \\
\hline$\geq 120($ ref. $<60)$ & $1.67(0.99-2.81)$ & & $0.64(0.15-2.65)$ & \\
\hline
\end{tabular}

$O R$ odds ratio, $C I$ confidence interval $p$ values less than 0.05 were bolded 
Table 3 Univariate and multivariate Cox regression analyses for overall survival

\begin{tabular}{|c|c|c|c|c|}
\hline \multirow[t]{2}{*}{ Variable } & \multicolumn{2}{|c|}{$\begin{array}{l}\text { Unmatched } \\
\text { Event/total }=3646 / 6888\end{array}$} & \multicolumn{2}{|c|}{$\begin{array}{l}\text { Matched } \\
\text { Event/total =1279/2408 }\end{array}$} \\
\hline & HR $(95 \% \mathrm{CI})$ & $p$ & HR $(95 \% \mathrm{CI})$ & $p$ \\
\hline \multicolumn{5}{|l|}{ Univariate } \\
\hline \multicolumn{5}{|l|}{ Age } \\
\hline $50-69$ (ref. < <0) & $1.01(0.92-1.12)$ & \multirow[t]{2}{*}{$<0.001$} & $1.05(0.89-1.24)$ & \multirow[t]{2}{*}{0.72} \\
\hline$\geq 70($ ref. $<50)$ & $1.25(1.13-1.39)$ & & $1.08(0.9-1.3)$ & \\
\hline \multicolumn{5}{|l|}{ Race } \\
\hline Black (ref. white) & $0.83(0.74-0.94)$ & \multirow[t]{3}{*}{$<0.001$} & $0.78(0.62-1)$ & \multirow[t]{3}{*}{0.24} \\
\hline Hispanic (ref. white) & $0.89(0.78-1.01)$ & & $0.98(0.79-1.21)$ & \\
\hline Asian/other (ref. white) & $0.73(0.6-0.88)$ & & $0.88(0.6-1.29)$ & \\
\hline \multicolumn{5}{|l|}{ Sex } \\
\hline Female (ref. male) & $0.84(0.78-0.91)$ & $<0.001$ & $0.78(0.68-0.91)$ & 0.001 \\
\hline \multicolumn{5}{|l|}{ Clinical T stage } \\
\hline 2 (ref. 1) & $1.06(0.9-1.24)$ & \multirow[t]{4}{*}{$<0.001$} & $1.1(0.86-1.39)$ & \multirow[t]{4}{*}{$<0.001$} \\
\hline 3 (ref. 1) & $1.24(1.08-1.43)$ & & $1.31(1.05-1.63)$ & \\
\hline 4a (ref. 1) & $1.51(1.24-1.86)$ & & $1.55(1.11-2.15)$ & \\
\hline 4b (ref. 1) & $2.36(1.64-3.39)$ & & $4.01(2-8.03)$ & \\
\hline \multicolumn{5}{|l|}{ Tumor location } \\
\hline Fundus/body (ref. cardia) & $0.86(0.77-0.96)$ & \multirow[t]{3}{*}{$<0.001$} & $0.8(0.61-1.05)$ & \multirow[t]{3}{*}{$<\mathbf{0 . 0 0 1}$} \\
\hline Antrum/pylorus (ref. Cardia) & $0.73(0.65-0.83)$ & & $0.69(0.51-0.94)$ & \\
\hline Overlapping (ref. cardia) & $1.33(1.16-1.53)$ & & $1.83(1.34-2.51)$ & \\
\hline \multicolumn{5}{|l|}{ Hospital setting } \\
\hline Comprehensive (ref. community) & $1.01(0.87-1.18)$ & \multirow[t]{3}{*}{$<0.001$} & $1.13(0.84-1.53)$ & \multirow[t]{3}{*}{0.46} \\
\hline Academic (ref. community) & $0.82(0.71-0.95)$ & & $1.03(0.77-1.37)$ & \\
\hline Other (ref. community) & $0.94(0.8-1.11)$ & & $1.09(0.79-1.5)$ & \\
\hline \multicolumn{5}{|l|}{ Year of diagnosis } \\
\hline 2011-2014 (ref. 2006-2010) & $0.93(0.87-1)$ & 0.04 & $0.92(0.82-1.04)$ & 0.18 \\
\hline Preoperative therapy & & & & \\
\hline CXRT (ref. chemotherapy) & $1.07(1-1.14)$ & 0.05 & $1.04(0.93-1.16)$ & 0.49 \\
\hline Radiation dose & & & & \\
\hline 40-60 Gy (ref. < 40 Gy) & $0.92(0.79-1.08)$ & 0.33 & $0.90(0.69-1.18)$ & 0.33 \\
\hline Radiation type & & & & \\
\hline Advanced (ref. conventional) & $0.98(0.9-1.08)$ & 0.74 & $0.94(0.80-1.11)$ & 0.47 \\
\hline Duration between chemo and surger & ays & & & \\
\hline 60-89 (ref. <60) & $0.78(0.66-0.92)$ & 0.001 & $0.84(0.61-1.17)$ & 0.62 \\
\hline $90-119$ (ref. <60) & $0.84(0.71-0.98)$ & & $0.87(0.63-1.2)$ & \\
\hline$\geq 120($ ref. $<60)$ & $0.91(0.77-1.08)$ & & $0.91(0.65-1.27)$ & \\
\hline Multivariate & & & & \\
\hline Age & & & & \\
\hline 50-69 (ref. <50) & $0.99(0.88-1.11)$ & $<\mathbf{0 . 0 0 1}$ & & \\
\hline$\geq 70($ ref. $<50)$ & $1.24(1.09-1.4)$ & & & \\
\hline Race & & & & \\
\hline Black (ref. white) & $0.84(0.72-0.98)$ & 0.003 & & \\
\hline Hispanic (ref. white) & $0.94(0.8-1.1)$ & & & \\
\hline Asian/Other (ref. white) & $0.68(0.53-0.86)$ & & & \\
\hline Sex & & & & \\
\hline Female (ref. male) & $0.84(0.76-0.92)$ & $<\mathbf{0 . 0 0 1}$ & $0.78(0.67-0.9)$ & 0.001 \\
\hline Clinical T stage & & & & \\
\hline 2 (ref. 1) & $1.01(0.86-1.18)$ & $<\mathbf{0 . 0 0 1}$ & $1.05(0.83-1.34)$ & $<0.001$ \\
\hline 3 (ref. 1) & $1.16(1.01-1.35)$ & & $1.26(1.01-1.58)$ & \\
\hline 4a (ref. 1) & $1.51(1.22-1.88)$ & & $1.5(1.07-2.1)$ & \\
\hline
\end{tabular}


Table 3 (continued)

\begin{tabular}{|c|c|c|c|c|}
\hline \multirow[t]{2}{*}{ Variable } & \multicolumn{2}{|c|}{$\begin{array}{l}\text { Unmatched } \\
\text { Event/total }=3646 / 6888\end{array}$} & \multicolumn{2}{|c|}{$\begin{array}{l}\text { Matched } \\
\text { Event/total =1279/2408 }\end{array}$} \\
\hline & $\mathrm{HR}(95 \% \mathrm{CI})$ & $p$ & $\mathrm{HR}(95 \% \mathrm{CI})$ & $p$ \\
\hline $4 \mathrm{~b}($ ref. 1$)$ & $2.54(1.73-3.72)$ & & $4.62(2.35-9.06)$ & \\
\hline \multicolumn{5}{|l|}{ Tumor location } \\
\hline Fundus/body (ref. cardia) & $0.89(0.77-1.03)$ & $<0.001$ & $0.83(0.64-1.09)$ & $<\mathbf{0 . 0 0 1}$ \\
\hline Antrum/pylorus (ref. cardia) & $0.74(0.63-0.88)$ & & $0.7(0.52-0.95)$ & \\
\hline Overlapping (ref. cardia) & $1.52(1.28-1.79)$ & & $1.87(1.37-2.56)$ & \\
\hline \multicolumn{5}{|l|}{ Hospital setting } \\
\hline Comprehensive (ref. community) & $1.05(0.87-1.27)$ & $<\mathbf{0 . 0 0 1}$ & & \\
\hline Academic (ref. community) & $0.89(0.74-1.06)$ & & & \\
\hline Other (ref. community) & $1.04(0.85-1.27)$ & & & \\
\hline \multicolumn{5}{|l|}{ Preoperative therapy } \\
\hline CXRT (ref. chemotherapy) & $1.05(0.96-1.15)$ & 0.33 & $1.03(0.92-1.15)$ & 0.63 \\
\hline \multicolumn{5}{|c|}{ Duration between chemo and surgery, days } \\
\hline $60-89$ (ref. $<60)$ & $0.75(0.62-0.92)$ & 0.001 & & \\
\hline 90-119 (ref. <60) & $0.82(0.67-0.99)$ & & & \\
\hline$\geq 120($ ref. $<60)$ & $0.89(0.73-1.09)$ & & & \\
\hline
\end{tabular}

$H R$ hazard ratio, $C I$ confidence interval

$p$ values less than 0.05 were bolded

$\mathbf{a}$

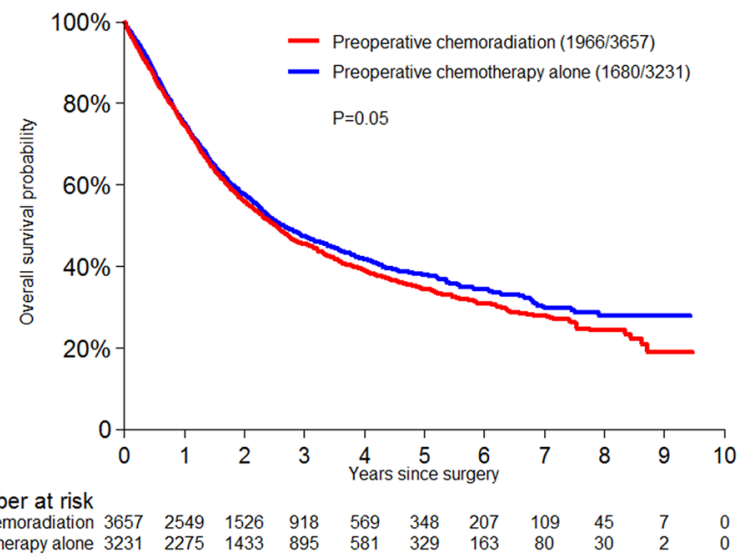

b

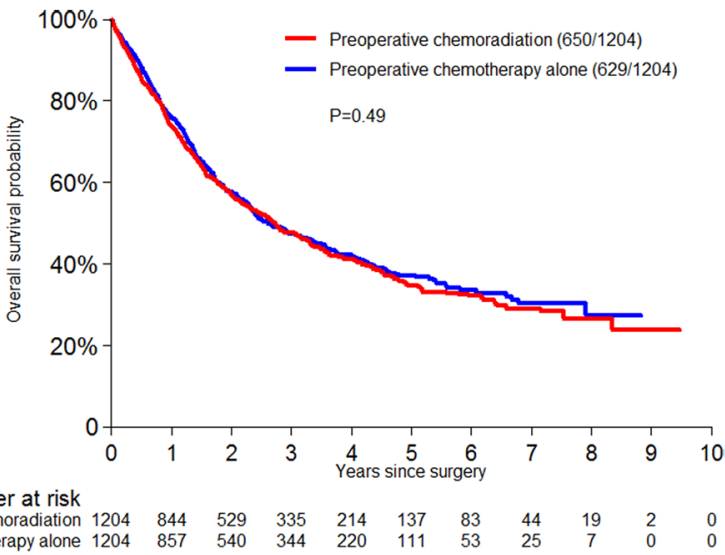

Fig. 1 Kaplan-Meier estimate for overall survival by radiation, a unmatched patients, $\mathbf{b}$ propensity score matched patients

\section{Short-term outcomes}

Among the 2408 matched patients, short-term outcomes were stratified by preoperative therapy regimen (Table 4). CXRT was not associated with increased 30-day mortality (2.8 vs. $2.9 \%$ ), readmission within 30 days ( 8.4 vs. $7.8 \%$ ), or length of surgical hospital stay (median, 9 vs. 9 days), compared with chemotherapy. Among 2376 patients with surgical margin status available, R0 resection was more frequently achieved in patients who underwent CXRT than in those who underwent chemotherapy [ $92 \%(1096 / 1,188)$ vs. 86\% (1025/1, 188); $p<0.001]$. Among 2368 patients with total number of lymph nodes examined by the pathologist after gastrectomy, patients who underwent CXRT had a lower mean number of examined lymph nodes [mean (standard deviation), 14.2 (9.2) vs. $19.2(11.7) ; p<0.001]$ and lower frequency of having at least 16 lymph nodes examined [39\% $(464 / 1,184)$ vs. $57 \%(671 / 1,184) ; p<0.001]$, compared with chemotherapy patients.

\section{Discussion}

In this retrospective cohort study of gastric cancer patients from the NCDB, preoperative CXRT was associated with a higher incidence of ypT0 as compared with those treated with chemotherapy only and was associated with a higher incidence of R0 resection; however, preoperative CXRT was not associated with improved OS. The safety profile 
Table 4 Short-term outcomes, stratified by preoperative therapy regimen

\begin{tabular}{|c|c|c|c|}
\hline & CXRT $(N=1204)$ & $\begin{array}{l}\text { Chemotherapy } \\
(N=1204)\end{array}$ & $p$ \\
\hline 30 day mortality $-N(\%), N=2390$ & & & 1.0 \\
\hline Yes & $34(2.8 \%)$ & $35(2.9 \%)$ & \\
\hline Readmission within 30 days $-N(\%), N=2324$ & & & 0.76 \\
\hline Yes & $98(8.4 \%)$ & $90(7.8 \%)$ & \\
\hline $\begin{array}{l}\text { Surgical inpatient stay, days from surgery-median } \\
\quad \text { (range), } N=2170\end{array}$ & $9(0-99)$ & $9(0-154)$ & 0.50 \\
\hline $\begin{array}{l}\text { Complete resection }(\mathrm{R} 0)-N(\%), N=2376 \\
\text { Yes }\end{array}$ & $1096(92 \%)$ & $1025(86 \%)$ & $<\mathbf{0 . 0 0 1}$ \\
\hline \multicolumn{4}{|l|}{ Number of lymph nodes examined, $N=2368$} \\
\hline Mean (standard deviation) & $14.2(9.2)$ & $19.2(11.7)$ & $<0.001$ \\
\hline Number $\geq 16-N(\%)$ & $464(39 \%)$ & $671(57 \%)$ & $<0.001$ \\
\hline
\end{tabular}

(e.g., mortality, readmission, and length of hospital stay) was equivalent between the CXRT and chemotherapy groups.

The CROSS trial showed a survival benefit of preoperative CXRT for patients with esophageal and esophagogastric cancers compared with the use of surgery alone. The study also showed a $29 \%$ incidence of pathologic complete response in the preoperative CXRT group [12]. Preoperative CXRT has also been used and studied for gastric cancer patients. Several phase II clinical trials that reported high incidences (20-30\%) of pathological complete response in gastric adenocarcinomas have shown the safety and efficacy of preoperative CXRT [13-15]. Radiation fields and technique used in preoperative CXRT vary by institution in the United States. In the international multicenter TOPGEAR trial [16], 45 Gy radiation therapy was delivered in 25 fractions to the entire stomach, any perigastric tumor extension, and regional lymph nodes using three-dimensional (3D) conformal techniques, intensity-modulated radiotherapy (IMRT), or volumetric-modulated arc radiotherapy (VMAT). However, such advanced radiation techniques are not available in all institutions and approximately $60 \%$ of patients in the current study underwent conventional radiation therapy (data not shown). The extent of radiation field also varies, but typically does not include extra-regional lymph nodes such as para-aortic lymph nodes or hepatoduodenal ligament lymph nodes unless found enlarged and suspected for metastasis. Based on reportedly high pathologic complete response rates, preoperative CXRT is believed to improve local control after gastrectomy, but the survival benefit of preoperative CXRT compared with chemotherapy alone is unknown in gastric cancer.

Our current study showed that preoperative CXRT is equivalent with respect to postoperative hospital stay and mortality compared to those receiving chemotherapy alone, a finding consistent with the interim results of the TOPGEAR trial [16] as well as our recent institutional report [17, 18].
The use of the NCDB enabled us to examine the outcomes of a large cohort of patients compared to the TOPGEAR trial, and the application of propensity score matching allowed us to balance the baseline characteristics of the two comparison groups. Therefore, the results of this study may serve as preliminary data, while the oncology community awaits the final reporting of the phase III randomized clinical trial. Our results identified a significantly higher incidence of ypT0 among CXRT patients, which likely contributes to improved local tumor control, although we were unable to evaluate this outcome in the current study because the NCDB does not collect data for recurrence variables.

Incidence of ypT0 was the main outcome of this study. We disregarded the field designation for N0 (ypTON0) in this categorization for three reasons. First, to increase the number of patients in the analyses (able to include patients with missing values for the $\mathrm{pN}$ category). Second, the quality and accuracy of $\mathrm{pN}$ staging may vary among institutions more significantly than pT staging. The $\mathrm{pN}$ category can also be biased by the extent of lymph node dissection and surgical technique, as well as the thoroughness of lymph node specimen assessment by pathologists [19]. Lastly, the number of lymph nodes examined can also be affected by the regimen of preoperative therapy, especially if radiation therapy was included. Indeed, the number of lymph nodes examined by pathologists was lower among patients who underwent CXRT than in the chemotherapy group in the current study, which is consistent with reports in colorectal cancer patients [20,21]; this may have decreased the sensitivity of identifying lymph node metastasis in the pathologic diagnosis and overestimated ypTON0 in the CXRT group. In contrast, accurate pT staging requires only gastrectomy and adequate pathologic review; ypT0 is a more robust outcome measure of pathologic response to preoperative therapy than ypN0. For the reasons noted, 
we considered ypT0 to be most effective and a reasonable outcome for comparing the effectiveness of CXRT and chemotherapy in the current study.

Selection bias is the major challenge when comparing the effectiveness of two different treatments in retrospective cohort studies using secondary data. For example, it is not feasible to assess the survival benefit of surgical resection of gastric cancer (or of any other solid organ malignancies), because the selection of patients for surgical resection cannot be reliably adjusted for by any statistical method. We consider that selection bias would be small in the selection between CXRT and chemotherapy for the preoperative therapy regimen for gastric cancer, which is mostly dependent on physician and institutional preference. However, we observed significant selection bias in this study by location of the tumor; CXRT was commonly used for cardia tumors while it was infrequently used for non-cardia tumors. It is also possible there remains residual confounding as the CXRT cohort may include patients with more advanced disease. Use of propensity score matching was conducted to decrease such selection bias and there was no OS difference by treatment regimen in the matched cohort. Using ypT0 as the primary outcome is a robust measure of treatment response, while OS can be affected by other factors such as patient performance status and comorbidities. We would postulate that a higher incidence of ypT0 seen in the CXRT group would have balanced the potential confounding, and the survival benefit of CXRT could have been underestimated.

Limitations of this study, other than potential selection bias as described above, include missing information and limited variables in the NCDB, which may have limited statistical adjustment and allowed residual confounding. The lack of recurrence variables in the data limits analyses to confirm the benefit of preoperative CXRT with respect to local disease control and disease-free survival. The variability of the preoperative therapy regimens administered within each cohort may have also affected survival outcomes of the study. There are several other variables of importance that are not included in the NCDB, such as site of positive surgical margin (distal, proximal, or radial margin), extent of radiation therapy field (whether whole stomach is radiated or not and whether radiation field includes extra-regional lymph nodes), and detailed regimens of chemotherapy with associated tolerance and completion rates. However, the strength of this study is a large number of study patients, carefully considered inclusion criteria, and appropriate use of statistical approaches. This study, to our knowledge, is the first to utilize the NCDB and propensity score matching to investigate the effect of CXRT and chemotherapy on the tumor response in gastric cancer.

\section{Conclusions}

This retrospective cohort study using a large national database showed a significantly higher incidence of ypT0 in gastric cancer patients who were treated with preoperative CXRT than in those treated with chemotherapy alone. Despite these encouraging results, the lack of OS benefit continues to support equipoise in ongoing clinical trials comparing these treatment regimens. Confirmation of the safety profile of preoperative CXRT also supports ongoing preoperative treatment in national guidelines while the results of the TOPGEAR trial are maturing.

Funding Supported in part by the National Institutes of Health under Award number P30CA016672 and used the Clinical Trials Support Resource.

\section{Compliance with ethical standards}

Conflict of interest The authors have no conflicts of interest to disclose.

Human/animal rights statement All procedures performed in studies involving human participants were in accordance with the ethical standards of the institutional and/or national research committee and with the 1964 Helsinki declaration and its later amendments or comparable ethical standards.

\section{References}

1. Macdonald JS, Smalley SR, Benedetti J, et al. Chemoradiotherapy after surgery compared with surgery alone for adenocarcinoma of the stomach or gastroesophageal junction. N Engl J Med. 2001;345:725-30.

2. Sakuramoto S, Sasako M, Yamaguchi T, et al. Adjuvant chemotherapy for gastric cancer with S-1, an oral fluoropyrimidine. N Engl J Med. 2007;357:1810-20.

3. Cunningham D, Allum WH, Stenning SP, et al. Perioperative chemotherapy versus surgery alone for resectable gastroesophageal cancer. N Engl J Med. 2006;355:11-20.

4. Ychou M, Boige V, Pignon JP, et al. Perioperative chemotherapy compared with surgery alone for resectable gastroesophageal adenocarcinoma: an FNCLCC and FFCD multicenter phase III trial. J Clin Oncol. 2011;29:1715-21.

5. Schuhmacher C, Gretschel S, Lordick F, et al. Neoadjuvant chemotherapy compared with surgery alone for locally advanced cancer of the stomach and cardia: European Organisation for Research and Treatment of Cancer randomized trial 40954. J Clin Oncol. 2010;28:5210-8.

6. Greenleaf EK, Hollenbeak CS, Wong J. Trends in the use and impact of neoadjuvant chemotherapy on perioperative outcomes for resected gastric cancer: evidence from the American College of Surgeons National Cancer Database. Surgery. 2016;159:1099-112.

7. Leong T, Smithers BM, Michael M, et al. TOPGEAR: a randomised phase III trial of perioperative ECF chemotherapy versus preoperative chemoradiation plus perioperative ECF chemotherapy for resectable gastric cancer (an international, intergroup 
trial of the AGITG/TROG/EORTC/NCIC CTG). BMC Cancer. 2015; 15:532.

8. American College of Surgeons. National Cancer Database. https ://www.facs.org/quality-programs/cancer/ncdb. Accessed 4 May 2018.

9. Amin MBES., Greene F, et al. AJCC cancer staging manual. 8th ed. New York: Springer; 2017.

10. Therneau TM, Grambsch P. Modeling survival data. New York: Springer; 2000.

11. Parsons LS. Performing a $1: \mathrm{N}$ case-control match on propensity score. In: Proceedings of the twenty-sixth annual SAS users group international conference. Montreal, Canada, 2004.

12. Shapiro J, van Lanschot JJ, Hulshof MC, et al. Neoadjuvant chemoradiotherapy plus surgery versus surgery alone for oesophageal or junctional cancer (CROSS): long-term results of a randomised controlled trial. Lancet Oncol. 2015;16:1090-8.

13. Ajani JA, Winter K, Okawara GS, et al. Phase II trial of preoperative chemoradiation in patients with localized gastric adenocarcinoma (RTOG 9904): quality of combined modality therapy and pathologic response. J Clin Oncol. 2006;24:3953-8.

14. Ajani JA, Mansfield PF, Janjan N, et al. Multi-institutional trial of preoperative chemoradiotherapy in patients with potentially resectable gastric carcinoma. J Clin Oncol. 2004;22:2774-80.

15. Ajani JA, Mansfield PF, Crane CH, et al. Paclitaxel-based chemoradiotherapy in localized gastric carcinoma: degree of pathologic response and not clinical parameters dictated patient outcome. J Clin Oncol. 2005;23:1237-44.

16. Leong T, Smithers BM, Haustermans K, et al. TOPGEAR: a randomized, phase III trial of perioperative ECF chemotherapy with or without preoperative chemoradiation for resectable gastric cancer: interim results from an international, intergroup trial of the AGITG, TROG, EORTC and CCTG. Ann Surg Oncol. 2017;24:2252-8.

17. Badgwell B, Ajani J, Blum M, et al. Postoperative morbidity and mortality rates are not increased for patients with gastric and gastroesophageal cancer who undergo preoperative chemoradiation therapy. Ann Surg Oncol. 2016;23:156-62.

18. Ikoma NDP, Blum M, et al.: Preoperative chemoradiation therapy does not increase risk of anastomotic leak in gastric cancer patients. Int J Radiat Oncol Biol Phys. 2017;99(3):660-6.

19. Morgan JW, Ji L, Friedman G, et al. The role of the cancer center when using lymph node count as a quality measure for gastric cancer surgery. JAMA Surg. 2015;150:37-43.

20. Kim HJ, Jo JS, Lee SY, et al. Low lymph node retrieval after preoperative chemoradiation for rectal cancer is associated with improved prognosis in patients with a good tumor response. Ann Surg Oncol. 2015;22:2075-81.

21. Damin DC, Rosito MA, Contu PC, et al. Lymph node retrieval after preoperative chemoradiotherapy for rectal cancer. J Gastrointest Surg. 2012;16:1573-80. 